TREATMENT

\title{
DCIS
}

https://doi.org/10.29289/259453942020V30S1066

\section{UNI-INSTITUTIONAL ANALYSIS OF PATIENTS DIAGNOSED WITH BREAST DUCTAL CARCINOMA IN SITU TREATED WITH BREAST-CONSERVING SURGERY AND ADJUVANT RADIOTHERAPY}

Felipe Rodrigues Costa Oliveira', Camila Zerbini Prata', Gil Facina', Roberto Araujo Segreto1, Rodrigo Souza Dias ${ }^{1}$ Universidade Federal de São Paulo - São Paulo (SP), Brasil.

Introduction: The treatment for Ductal Carcinoma in situ (DCIS) can be mastectomy, breast-conserving surgery with or without adjuvant Radiotherapy (RT). Regarding the conserving treatment, ipsilateral recurrence is the main reason of concern. Randomized studies show that RT reduces the risk of local recurrence (LR). Age, comedonecrosis, absence of endocrine therapy (ET) and positive margins are also prognostic factors. Objective: To assess the local relapse-free survival (LRFS) of patients with DCIS treated with breast-conserving surgery, followed by adjuvant RT, and to identify possible prognostic factors related to LR. Method: between March 2007 and December 2017, we identified 95 women diagnosed with DCIS and treated with breast-conserving surgery and adjuvant RT in Hospital São Paulo (HSP/UNIFESP). Regarding RT, we used the 3D technique with conventional fractionation (50/50.4 Gy and 2/1.8 Gy fraction per day), or hypofractionation (40 Gy and 2.67 Gy fraction per day). The data were submitted to descriptive analysis and evaluation of LR. Factors such as characteristics of the patients, the tumor and the treatment were correlated by using Fisher's Exact Test and Kruskall-Wallis test. The log-rank test was used for the Kaplan-Meier comparison. The results were considered as statistically significant when $\mathrm{p}<0.05$. Results: $71.6 \%$ of the patients were 50 years old or older; margins were negative in $70.5 \%$ of the cases, and $78.9 \%$ of the patients presented with positive hormone receptors. Regarding RT, $89.5 \%$ of the patients were treated with conventional fractionation. The median follow-up of patients was 67 months (8-150 months). LRFS was $97.8 \%$ and $91.9 \%$ in 5 and 10 years, respectively. Among the assessed factors, negative hormone receptors $(\mathrm{p}<0.001)$ and absence of ET ( $\mathrm{p}=0.022)$ were prognostic factors for LR. The margin status was not associated with higher rates of LR. Conclusion: The LRFS of the patients diagnosed with DCIS who underwent a conserving treatment in HSP/ UNIFESP is favorably compared to the results described in the literature. Negative hormone receptors and absence of ET had an influence on local control. 